\title{
Non-Celiac Consumer Knowledge Regarding Gluten-free Diets
}

\section{Andrew Hou ${ }^{1}$, Helen Heacock ${ }^{2}$}

1. Lead Author and Researcher, B. Tech Student, School of Health Sciences, British Columbia Institute of Technology, 3700 Willingdon Ave, Burnaby, BC V5G 3H2

2. Supervisor, School of Health Sciences, British Columbia Institute of Technology, 3700 Willingdon Ave, Burnaby, BC V5G 3H2

\begin{abstract}
BACKGROUND: With the rising trend in gluten-free diets, it is imperative that there is high consumer product literacy so that the public makes informed decisions in regards to their diet and health. Knowledge taken from reputable sources and recognizing unsubstantiated health claims regarding gluten-free diets is critical for a non-celiac consumer.

METHODS: A survey was used to investigate why non-celiac consumers elect to follow gluten-free diets and why they believe that the elimination of gluten from their diet is healthy. This project also tested consumer knowledge regarding gluten.

RESULTS: During a 2 month period, total of 376 individuals participated in the survey. Only 322 participants fell under the inclusion criteria of this study. Women who elected to participate in gluten-free diets (but did not have Celiac’s Disease themselves) had higher overall test scores and men in the general population had lower overall test scores $(\mathrm{p}=0.000017)$.
\end{abstract}

CONCLUSIONS: Based on overall test scores and percentages of correct responses for specific questions, there seems to be deficiencies in both the average consumer and non-celiac-gluten-avoider-consumer knowledge regarding gluten, gluten-free products and diets

KEYWORDS: Survey, gluten, gluten-free, gluten-free diets, non-celiac gluten sensitivity

\section{Introduction}

Over the past few years, there has been a rise in the popularity of gluten-free products. Currently, it is not uncommon to see sections devoted to gluten-free foods at a local supermarket while several years ago, one would have to seek out a specialty store.

Perplexingly, there is little information available about the benefits of a gluten-free diet for a non-celiac consumer. All information available appears to come from either anecdotal stories from gluten-free-nonceliac advocates or from questionable sources.
The purpose of this study was to survey the public consumer's perception on the still rising trend of gluten and gluten-free products, specifically with non-celiac consumers.

This project sought to discover why non-celiac consumers elected to follow a gluten-free diet and why they believed that the elimination of gluten from their diet was healthy. This project also sought to test consumer knowledge about gluten. 


\section{Literature Review}

\section{Gluten}

Gluten is a protein composite of gliadin and glutenin that is found in certain grain species. Section B.01.010.1(1) of the Canadian Food and Drug Regulations state that gluten is any gluten protein that is derived from wheat, barley, oats, rye, triticale, kamut or spelt. In addition, any gluten proteins that are modified or fractioned from the above listed cereals are to be considered gluten in Canada (Food and Drug Regulations (C.R.C., C. 870) (a), 2013).

Gluten is widely used in processed foods due to its ability to make bread and baked-good products bind and prevent crumbling (Canadian Celiac Association , 2011a). Thus, gluten is commonly associated with bread products. But it is present in other foods, such as soy sauce, salad dressings, potato chips, Eucharistic wafers, hot dogs and even vodka. Gluten is also present in a variety of non-food products, such as toothpaste, makeup, medicine and even playdough (Gulli, 2013).

\section{Celiac Disease}

Celiac disease (CD), also known as coeliac or celiac sprue disease, is an autoimmune disorder that affects the mucosal lining of the small intestine. The absorptive surface is damaged by gluten (specifically a reaction to the protein gliadin), resulting in the body's inability to properly absorb nutrients. It affects approximately $1 \%$ of the Canadian population; it is estimated that 1 in 133 Canadians (approximately 340,000 Canadians) are affected by CD (Canadian Celiac Association , 2011a).

\section{Symptoms of Celiac Disease}

$\mathrm{CD}$ has a variety of nonspecific symptoms, such as chronic diarrhea, weight loss, fatigue, anemia, cramps, bloating, irritability and malnutrition. The severity of symptoms varies from patient to patient. Some patients may be asymptomatic, but damage to the intestinal lining may still occur (Canadian Celiac Association , 2011a).

\section{Diagnosis of Celiac's Disease} Diagnosis of CD must be confirmed with a biopsy of the duodenal-jejunal mucosal lining to determine if lesions from gluten damage have occurred (Villanacci \& Corazza,
2005). Preliminary screening may be conducted by a physician using clinical signs and blood tests to select and screen patients. (Canadian Celiac Association , 2011a).

Available information about Celiac Disease $\mathrm{CD}$ has been extensively studied. Some aspects of the immunopathogensesis, prevalence, mortality and genetic host factors that affect host susceptibility to $\mathrm{CD}$ are understood. However, there are significant areas of information missing that would give understanding of its full mechanism (Kagnoff, 2007).

It is believed that the ratio for diagnosed to undiagnosed CD patients is 1:8.6 (Gulli, 2013). A study found that undiagnosed $\mathrm{CD}$ leads to a four-fold increase in the risk of untimely death due to health complications and that the prevalence of undiagnosed $\mathrm{CD}$ appears to have increased in the last 50 years (Rubio-Tapia, et al., 2009). However, the increased prevalence may be more likely to public awareness of CD, varying severity of symptoms and the development of more sensitive diagnostic methods.

\section{Treatment of Celiac Disease}

There is no known cure for CD. It can, however, be effectively controlled and treated with an adherence to a lifelong gluten-free diet, allowing the intestines to heal. There are numerous pieces of literature that prove the benefits of a gluten-free diet for a CD consumer (Canadian Celiac Association, 2011a).

\section{Non-Celiac Gluten Avoidance}

Recently, there has been a rise in a number of consumers who self-diagnose themselves as having a non-celiac gluten sensitivity (NCGS), where a person experiences the same symptoms of a celiac patient after gluten consumption, but does not have CD themselves (Gulli, 2013).

The Canadian Celiac Association uses an umbrella term called gluten sensitivity to describe NCGS. The "term is used to describe patients who get a variety of symptoms when they eat gluten and feel better on a gluten-free diet but do not have celiac disease" (Canadian 
Celiac Association, 2011b). Intestinal biopsies of such patients are normal and show no signs of damage.

In the existing literature available, there is little conclusive evidence that demonstrates NCGS to be a medically prescribed condition. There also is little consensus in the medical community if it is a medically prescribed condition. A study conducted in New Zealand found that gluten avoidance in non-celiac consumers seemed to be 5 times more common than a medically diagnosed CD consumer (Tanpowpong, et al., 2012). It is believed there are 7 million gluten avoiders in Canada who do not have CD or have any medically prescribed reason to avoid gluten (Gulli, 2013).
There are mixed views of NCGS within the scientific community, with some doubting its existence at all. The results of a 2011 study suggest that CD and NCGS are distinctly different conditions with distinct intestinal mucosal responses to gluten (Sapone, et al., 2011). A 2012 paper by Sapone et al. suggested that there could be 3 types of reactions to gluten and that there may be a wider spectrum of gluten-related disorders than previously thought (Sapone, et al., 2012). The table below illustrates the differences concluded in Sapone's 2012 study:

\begin{tabular}{|c|c|c|c|}
\hline & Celiac Disease & Wheat Allergy & Non-Celiac Gluten Sensitivity \\
\hline $\begin{array}{c}\text { Disease } \\
\text { Description }\end{array}$ & $\begin{array}{l}\text { Autoimmune disorder in } \\
\text { reaction to the } \\
\text { consumption of gluten. } \\
\text { Diagnosis requires positive } \\
\text { results on serum } \\
\text { antitransflutaminase or } \\
\text { antiendomysial antibody } \\
\text { testing and small-bowel } \\
\text { villous flattening }\end{array}$ & $\begin{array}{l}\text { Allergic response to gluten. } \\
\text { Cross-linking of } \\
\text { immunoglobulin } \operatorname{Ig}(\mathrm{E}) \text { by repeat } \\
\text { sequences in gluten peptides } \\
\text { that triggers release of chemical } \\
\text { mediators, such as histamine, } \\
\text { from basophils and mast cells. }\end{array}$ & $\begin{array}{l}\text { Patient inability to tolerate gluten. } \\
\text { Development of an adverse reaction } \\
\text { after consumption of gluten that does } \\
\text { not lead to intestinal damage. } \\
\text { Self-diagnosed. }\end{array}$ \\
\hline Mechanism & \multicolumn{2}{|c|}{ Reaction to gluten mediated by T-cell activation in GI mucosa } & $\begin{array}{l}\text { Unknown. Neither allergic or } \\
\text { autoimmune mechanisms are } \\
\text { involved }\end{array}$ \\
\hline
\end{tabular}


Below is a table taken from a study conducted in 2012 by DiSabatino and Corazza.

\section{Table 1. Main Characteristics Distinguishing Celiac Disease From Nonceliac Gluten Sensitivity}

\begin{tabular}{|c|c|c|}
\hline Characteristic & Celliac Disease & Noncellac Gluten Sensitivity \\
\hline Synonyms & Celiac sprue, gluten-sensitive enteropathy & $\begin{array}{l}\text { Noncellac gluten intolerance, nonceliac wheat intolerance, gluten } \\
\text { sensitivity, gluten hypersensitivity }\end{array}$ \\
\hline Prevalence & About $1 \%$ of the general population & Unknown, but suspected to be higher than that of celiac disease \\
\hline Cenetics & Related to HLA-DQ2 or HLA-DQ8 & Unrelated to a specific HLA haplotype \\
\hline Mechanisms & $\begin{array}{l}\text { Predominant adaptive immune reaction to } \\
\text { gluten peptides restricted by HLA-DQ2 } \\
\text { or HLA-DQ8 }\end{array}$ & $\begin{array}{l}\text { Unknown but multiple mechanisms are suspected, including innate immune } \\
\text { reaction to gluten; IgE-mediated wheat allergy; starch carbohydrate } \\
\text { malabsorption; opioid-like activity of gluten; gluten-induced, low-grade } \\
\text { inflammation; and nocebo effect of gluten-containing food }\end{array}$ \\
\hline Serum antibodies & $\begin{array}{l}\text { Positive results on TTA, EMA, or ACA } \\
\text { testing }\end{array}$ & $\begin{array}{l}\text { Negative results on TTA and anti-EMA testing, sometimes positive results } \\
\text { on IgG AGA testing }\end{array}$ \\
\hline Villous flattening & Present & Absent \\
\hline Symptoms & Intestinal and extraintestinal & Intestinal and extraintestinal \\
\hline Morbidity & Increased & No data \\
\hline Mortality & Increased & No data \\
\hline
\end{tabular}

$\mathrm{AGA}=$ antigliadin antibodies; $\mathrm{EMA}=$ endomysial antibodies; $\mathrm{TTA}=$ antitransglutaminase antibodies.

Both studies by Sapone and DiSabatino/Corazza sought to create some sort of criteria to define NCGS. However, the ambiguity and missing pieces of information in both studies shed little light on the matter.

A study by Holmes in 2013 suggested that “non-coeliac gluten intolerance affected some patients and could be successfully treated by diet but the mechanisms responsible for producing ill-health were unknown” (Holmes, 2013). There is no diagnostic test currently available to detect or validate this condition in patients; a person with NCGS conducts a self-diagnosis by recognizing an abatement of symptoms when they avoid gluten. To further complicate matters, the symptoms of NCGS are generic and overlap with symptoms commonly seen in irritable bowel syndrome (Holmes, 2013).

Overall, current literature illustrates that there is little information available in regards to NCGS. Some physicians and researchers believe that NCGS may exist in patients. However, the lack of consensus on defined diagnostic criteria makes it difficult to know how prevalent it is within the population (DiSabatino \& Corazza, 2012).

\section{Gluten-Free Products}

A gluten-free product would imply to a consumer that the product is formulated with a complete absence of gluten.

However, the Codex Alimentarius states that a gluten-free product must not have a gluten content that exceeds 20 ppm (Codex Alimentarius, 2008). Some countries have specific gluten-free labeling regulations dictating allowable gluten content while other countries do not. Section B.24.018 of the Canadian Food and Drug Regulations states that for a product to be declared gluten-free, it must "Not contain any gluten protein or modified gluten protein, including any gluten protein fraction” (Food and Drug Regulations (C.R.C. C. 870) (b), 2013). In addition, Health Canada considers any product that contains less than 20 ppm of gluten (that was introduced into the product from cross-contamination) to meet the intent of the Food and Drug Regulations and said product can be marketed as gluten free (Health Canada, 2012).

The threshold level of 20 ppm was chosen in Canada as current evidence indicates that this would protect the health of the vast majority of CD patients. Furthermore, with new labeling regulations introduced by Health Canada in 2011, any intentionally added gluten 
sources must be declared on the ingredient list if the gluten content is $10 \mathrm{ppm}$ or higher for all prepackaged food products (Health Canada, 2012).

There is no internationally accepted standard for an allowable limit of gluten in a gluten-free product. The United States follows the same limit and principle as the Codex and Canada. Some European countries and New Zealand/Australia differentiate between gluten-free and low-gluten products, with differing threshold limits for each (Diaz-Amgio \& Popping, 2012).

There also is discourse within the medical community about acceptable levels of gluten in a glutenfree product. While some studies find that $20 \mathrm{ppm}$ should be the maximum allowable limit, others argue that a gluten content of 100 ppm is still safe (Collin, Thorell, Kaukinen, \& M'aki, 2004).

\section{Increase in the popularity of gluten-free products}

The gluten free industry in Canada was valued $\$ 90$ million last year and is expected to have a $10 \%$ growth each year until 2018. On average, gluten-free products are 242-455\% more expensive than non-gluten free products (Gulli, 2013). The market retail sales in 2010 reached approximately $\$ 2.6$ billion US for gluten-free foods and beverages (Worosz \& Norbert, 2012).

\section{Rise in Non-Celiac Gluten Avoidance}

DiSabatino and Corazza speculated that "general public awareness of NCGS...[is] higher than that of celiac disease.” In February 2012, the ratio between Google and PubMed citations for non-celiac gluten sensitivity was approximately 4600 to 1 , and NCGS was searched ten times more than breast cancer, Alzheimer's, lung cancer or even celiac disease itself (DiSabatino \& Corazza, 2012).

Gluten-free websites and advocates claim that a gluten-free lifestyle has a range of health benefits. An article in the BC Medical Journal notes the rise in the NCGS health trend. The authors found that the reported health claims were that gluten-free diets for non-celiacs can cure diabetes, obesity, rheumatic illnesses and cataracts. However, the authors note that these health claims are unsubstantiated by any studies (Cadenhead \& Sweeny, 2013).

There also is a popular perception that a glutenfree diet will lead to weight loss and an overall healthier diet. Mintel, a private market research group, released a privately conducted study focusing on consumer perceptions of gluten-free foods in the United States. The databases and reports were only available to subscribing clients but the limited information released stated that " $65 \%$ of consumer[s] who eat or used to eat gluten-free foods do so because they think they are healthier and $27 \%$ eat them because they feel [it] aid[s] in their weight loss efforts” (Mintel, 2013).

However, numerous dietitians say that glutenfree processed foods are often lower in fiber and nutrients (such as calcium, vitamin D and folate) than their gluten counterparts. Gluten-free products often have substitute starches, such as rice and tapioca flour, that have a higher glycemic index than wheat flour. These products often can have higher fat and lower protein content than their counterparts due to these substitutes. An article in Maclean's magazine suggests that the benefit of perceived weight loss may be due to the elimination of fast/processed foods and refined grains from the person's diet rather than the elimination of gluten. The author suggests that the weight loss is not from the exclusion of gluten, but may be because overall, the consumer is eating a healthier, balanced diet (Gulli, 2013)

\section{Labeling Inaccuracies}

Purity of gluten-free products is a necessity for CD patients. An article from the Journal of Consumer Affairs suggests that the "current [market] trajectory of glutenfree [products] is likely to expose consumers living with CD...to greater [health] risks.” The authors caution that "with [an] increasing consumer demand, new firms entering the gluten-free industry may not be able to produce acceptable products for their consumers, leading to product purity and literacy being severely compromised” (Worosz \& Norbert, 2012). 
In 2002, a study found that $20 \%$ of products marketed at the time as gluten-free contained 22-71ppm of gluten (Lardizabal, Lynn, Niemann, Sue, \& Hefle, 2002). In 2008, the Chicago Tribune carried out a product survey and found that several Wellshire Kid's brand gluten-free products contained 116-2200 ppm of gluten (Roe, 2008).

Not only is this deceptive to the general public, this is also dangerous to a CD/NCGS consumer. There is consumer dependency upon the processor to produce a safe product as there is no worldwide standard for glutenfree products. Competent product literacy is a necessity as a CD/NCGS consumer, but the Journal of Consumer Affairs article warned that "most consumers do not know the amount of gluten in a product labeled gluten-free at the point of purchase, nor do they know the amount of gluten in the product after consumption.” The article suggests that an active consumer (ie: one who is genuinely concerned about their consumption of gluten and does not follow a fad/trend) would "seek out safe products...mak[ing] an effort to attain greater product literacy” (Worosz \& Norbert, 2012).

\section{$\underline{\text { Relation to Public Health }}$}

Currently, there is conflicting information regarding gluten-free diets. The scientific and medical community asserts that the health claims of a gluten-free lifestyle for a non-celiac consumer are currently unsubstantiated by any scientific study. Numerous dietitians and physicians say that gluten-free diets often are lower in essential nutrients and have higher caloric content than their nongluten free counterparts (Gulli, 2013). Gluten-free diet advocates swear of overall healthier lifestyles and abatement of chronic disease symptoms. If there really is no benefit for a non-celiac consumer on a gluten free diet, then there seems to be a communication barrier between the evidence (or lack therefore) from scientific studies to the consumer.

As such, because of the conflicting information, this study would be an opportunity to investigate what health benefits consumers believe they gain from following a gluten-free diet. If misconceptions are identified, actions could be taken to rectify and expose the falsehood in those misconceptions.

Testing consumer knowledge of gluten would be an ideal way to determine consumer product literacy, the likelihood of consumer misunderstanding and additional factors that require further investigation.

The results of this study could be of interest to primary health care providers (ie: nurses, dieticians and physicians) so they could be aware of misconceptions and questions that they would receive from their patients. However, the knowledge gained from this study could also be applicable to other professions, such as an Environmental Health Officer (EHO).

The primary role of an EHO is to protect the public health through education, followed by progressive enforcement when necessary. While knowledge regarding gluten-free diets does not fall under the traditional jurisdiction of EHOs (ie: pools and food premise inspections), the core principle of education is still the same and equally important. It is be possible that an EHO would be asked about gluten-free diets given the multidisciplinary nature of the profession. Also, EHOs are becoming much more involved in health promotion and education along with planning healthy built environments (where food security and nutrition are key topics). And finally, as of 2009, EHO's in British Columbia are mandated to ensure compliance to the $\mathrm{BC}$ Trans Fat Regulation (another practice that goes beyond the traditional scope of inspections).

Therefore, understanding the reasoning behind the popularity of gluten free diets could be important for an EHO. 


\section{Materials and Methods}

\section{Description of Standard Methods}

The data collection period started on January $16^{\text {th }}$, 2014 and ended March $10^{\text {th }}$, 2014. The study was conducted using data collected from an online self-administered survey that used standardized close-ended questions on Survey Monkey (SurveyMonkey, 2013). The survey was accessible for free to the public and was separated into 3 distinct sections.

The first section of the survey covered questions regarding age, gender, ethnicity, education level, celiac or non-celiac status, income level and informed consent. The first section had the main exclusion factor that this study used to determine participant eligibility.

The second section of the survey had glutenknowledge testing questions. The correct answers to the questions had a corresponding score and the scores were used in the statistical analysis to determine relationships, if any, in participant knowledge with corresponding factors. The participants were not informed that they were being scored, of how the scoring system worked, nor were they notified of what their scores were.

Questions 8 to 14 were used to evaluate consumer gluten knowledge and were used to calculate test scores. Each question was given a score of 1, for a total of 7 points overall.

The third and final section of the survey focused on the perceived health benefits of gluten-free diets. Participants were asked what the main perceived health benefit they felt they would gain from following a gluten-free diet.

A link to the questionnaire was distributed using Facebook and the researchers email list (Facebook, 2014). In both cases, the researcher encouraged participants to further distribute the survey to other respondents. Emails followed a standardized format. A reminder email was sent out on the $15^{\text {th }}, 30^{\text {th }}$ and $45^{\text {th }}$ days of the data collection period.

Excel was used for data manipulation (Microsoft Office, 2007). NCSS was used for statistical analysis (Hintze, 2013).

\section{Inclusion and Exclusion Criteria}

Any individual, with the exception of those who were medically diagnosed with Celiac's disease, was eligible to participate in this study. The survey had a question that explicitly asked participants if they had been medically diagnosed for Celiac's disease by a physician, as this is the only definitive method to fully diagnose the disease (Canadian Celiac Association, 2011).

Those who were under the age of 16 were excluded as the researcher felt that it was be unethical for them to participate as they were below the age of consent. The researcher also excluded any data from respondents who did not properly complete questions that verified participant year of birth and age.

\section{Ethical Considerations}

This survey involved human subjects. Therefore, it was subject to following the BCIT Research Ethics for Human Participants (British Columbia Insitute of Technology, 2013).

Issues of beneficence and autonomy were thoroughly examined. Informed, voluntary consent was obtained prior to the participants taking the survey. Notification of participant confidentiality and anonymity, along with notification that the participant could withdraw from survey at any point without consequences, was clearly stated prior to participants taking the survey. Any participant who did not agree to the confidentiality terms or did not provide consent to participate in the study was immediately considered ineligible and could not participate in the survey.

Contact information of the researcher was included for any participants if they wished to have any questions or concerns addressed.

Participants were not informed of the scoring system for the Knowledge Testing section of the survey. This was done to ensure that participant answers were 
truthful and had no motivation to research answers prior to responding.

The study was thoroughly examined by the BCIT Environmental Health Research Advisors prior to commencement to ensure there were no unaddressed ethical considerations

\section{Results}

A total of 376 individuals participated in the survey. Only 322 participants fell under the inclusion criteria of this study. A summery table of the number and percentage of participants for several parameters of interest is show by the figure below.

\section{Categorical Observations}

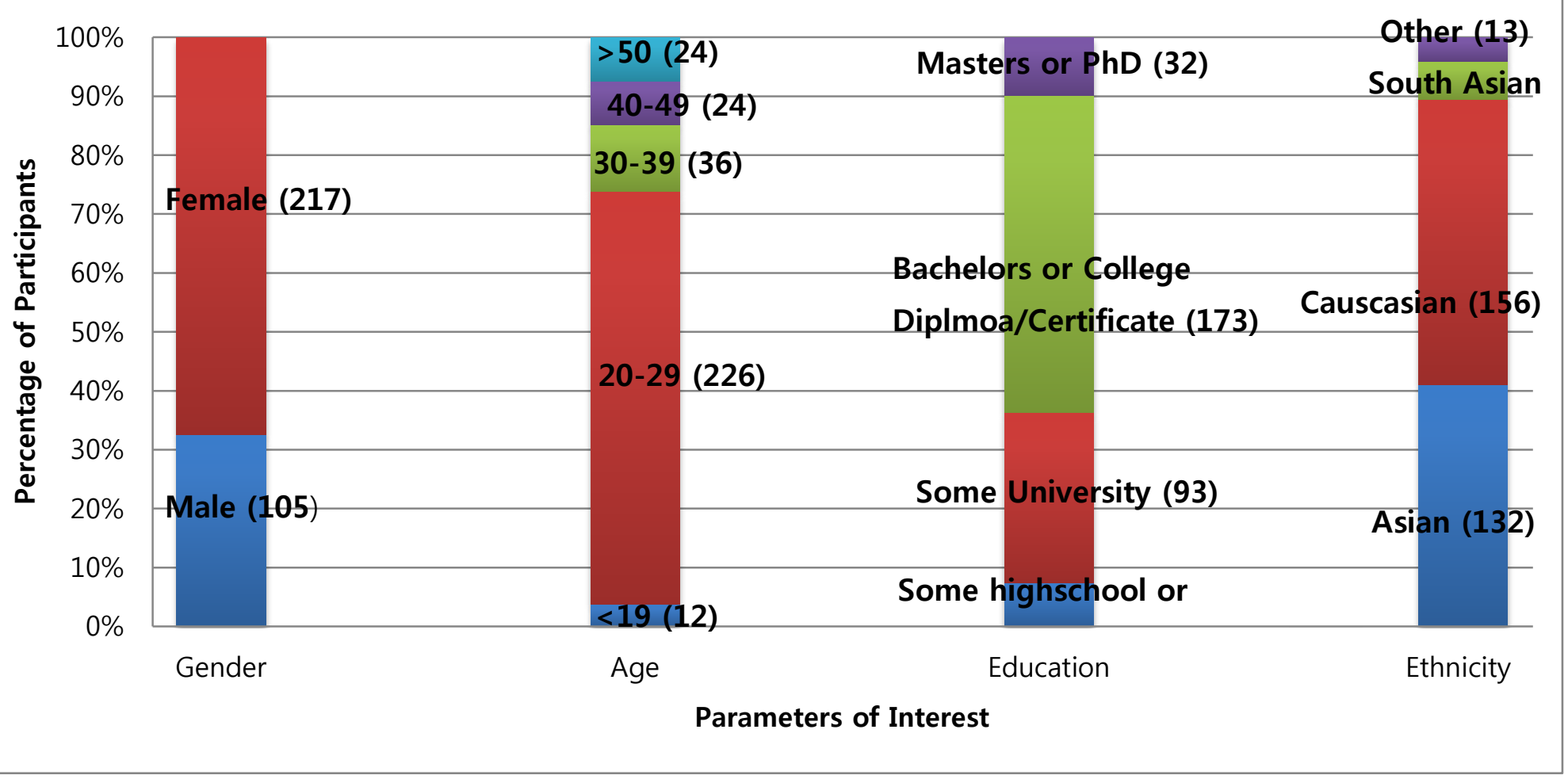

Figure 1: Summery of Categorical Observations

46 respondents chose not to answer the question regarding annual income. Of those that did respond, 48\% had an annual income less than \$15,000; $15 \%$ had an income ranging between \$15, 000 and \$29, 999; 14\% between \$30, 000 - \$44, 999; 8.7\% between \$45, 000, and \$59, 999; 8\% between \$60,000 - \$74, 999; 1.5\% between $\$ 75,000$ - \$89,999; and 5\% over \$ 90,000

The charts below summarize the reasons why participants followed a gluten-free diet and their frequency in following a gluten-free diet. 317 participants chose to answer these questions. 


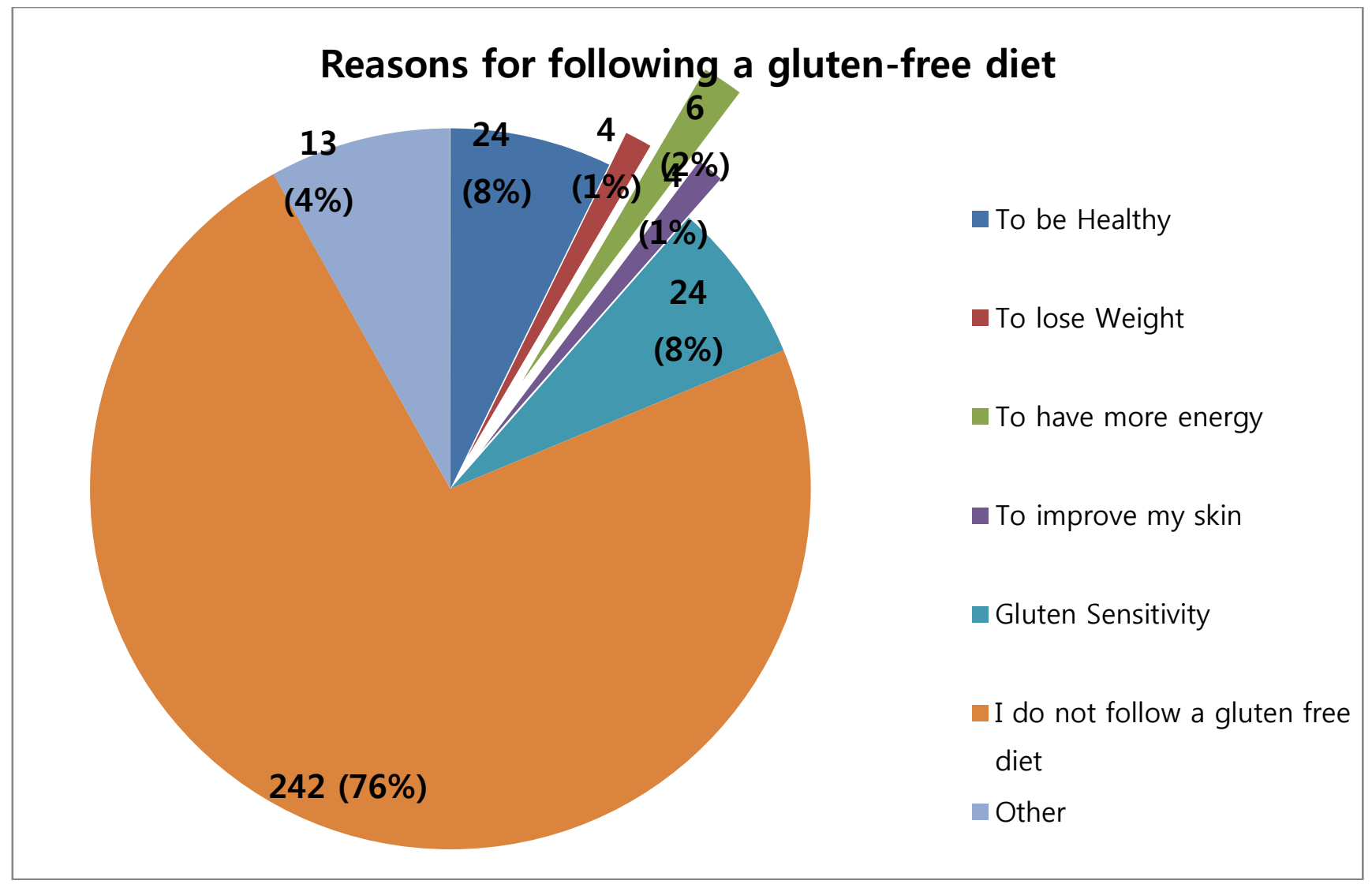

Figure 2: Summery of Participants Reasons for following gluten-free diets

\section{Frequency in following a gluten-free diet}

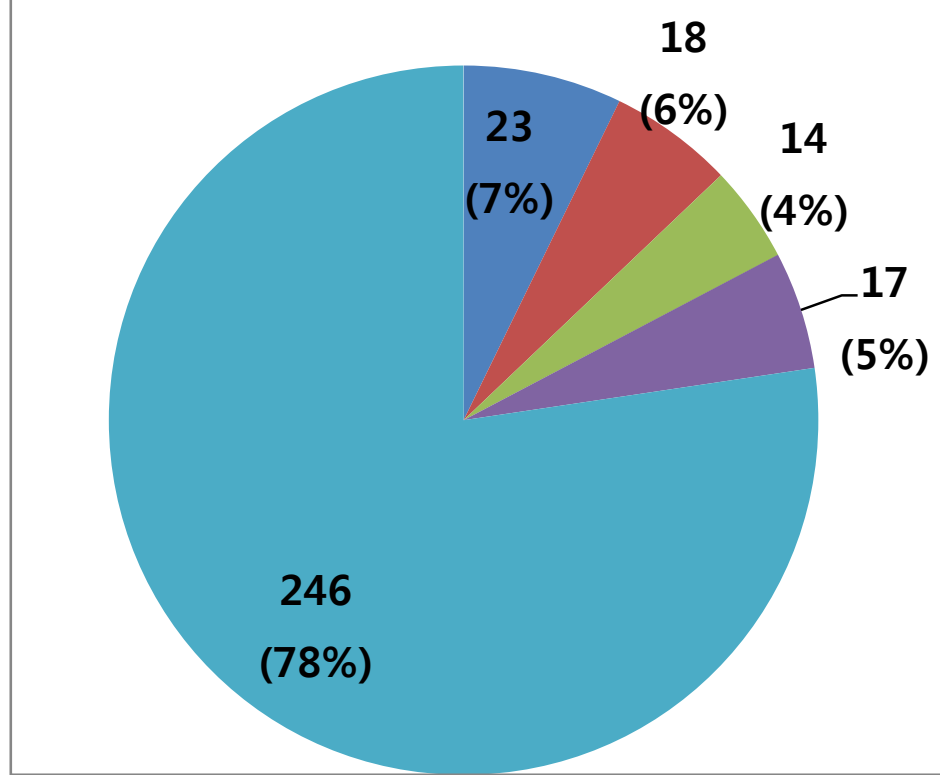

All the time. I only eat foods that are gluten-free

Frequently, 3-5 times a week

Often, 1-2 times a week

Rarely, only once or twice a month

I do not follow a gluten-free diet

Figure 3: Summery of Participants Frequency in following gluten-free diets 
Of the total respondents, 72 participants fell into the category of "non-celiac gluten avoiding consumers" (NCGAC). These participants declared that they elected to follow a gluten-free diet. The other 250 participants (who did not follow a gluten-free diet) were categorized as the "general public."

The major sources of information regarding gluten-free diets for consumers were their friends, internet sites and magazines.

\section{Test Scores}

For the gluten-knowledge test, the total score was out of 7 . The mean score for all respondents was 3.22 (46\%). The mean score for the general public was 3.01 (43\%) and the mean score for NCGAC was 3.92 (56\%).

From the knowledge testing questions, it was found for NCGACs that only:

- $55.6 \%$ of respondents knew that gluten is composed of glutenin and gliadin

- $27.8 \%$ and $32 \%$ of respondents knew that triticale and kamut are grains that contain gluten

- $51.4 \%$ of respondents knew that the symptoms caused by non-celiac gluten sensitivity are similar to those caused by CD.

- $38.9 \%$ of respondents knew that licorice contains gluten
- $41.7 \%$ of respondents knew that gluten-free processed foods often have higher calories and lower nutritional content than their gluten counterparts

- $23.6 \%$ of respondents knew the regulatory body that regulates gluten-free products in Canada.

In contrast, from the knowledge testing questions, it was found for the general public that only:

- $44.4 \%$ of respondents knew that gluten is composed of glutenin and gliadin

- $17.6 \%$ and $14.8 \%$ of respondents knew that triticale and kamut are grains that contain gluten

- $31.2 \%$ of respondents knew that the symptoms caused by non-celiac gluten sensitivity are similar to those caused by CD.

- $18 \%$ of respondents knew that licorice contains gluten

- $15.6 \%$ of respondents knew that gluten-free processed foods often have higher calories and lower nutritional content than their gluten counterparts

- $27.6 \%$ of respondents knew the regulatory body that regulates gluten-free products in Canada

The table below summarizes the test scores.

Table 1: Descriptive Statistical Summary of Test Scores for All Respondents, General Public and NCGAC

\begin{tabular}{|c|c|c|c|}
\hline & All Respondents & General Public & $N C G A C$ \\
\hline Mean & 3.22 & 3.01 & 3.92 \\
\hline Standard Error & 0.08 & 0.09 & 0.17 \\
\hline Median & 3.24 & 2.96 & 3.85 \\
\hline Mode & 1.48 & 1.48 & 4.71 \\
\hline $\begin{array}{l}\text { Standard } \\
\text { Deviation }\end{array}$ & 1.42 & 1.35 & 1.44 \\
\hline $\begin{array}{l}\text { Sample } \\
\text { Variance }\end{array}$ & 2.02 & 1.83 & 2.07 \\
\hline Kurtosis & -0.55 & -0.48 & -0.56 \\
\hline Skewness & 0.02 & 0.03 & -0.28 \\
\hline Range & 6.86 & 6.67 & 6.55 \\
\hline Minimum & 0.00 & 0.00 & 0.31 \\
\hline Maximum & 6.86 & 6.67 & 6.86 \\
\hline Sum & 1035.29 & 753.14 & 282.14 \\
\hline Count & 322 & 250 & 72 \\
\hline
\end{tabular}


The table below summarizes the results of the statistical analysis:

Table 2: Summary of Hypothesis Testing

\begin{tabular}{|l|l|l|l|}
\hline Hypothesis & Statistical Test & P-value & Accept/Reject \\
\hline Gender and Reason (Ho1) & Chi Squared & $\mathrm{p}=0.43$ & Accept Ho1 \\
\hline Gender and Test Scores (Ho2) & ANOVA & $\mathrm{p}=0.00$ & Accept Ha2 \\
\hline Education and Test Scores (Ho3) & ANOVA & $\mathrm{p}=0.70$ & Accept Ho3 \\
\hline General Public vs NCGAC Test Scores (Ho4) & T-test & $\mathrm{P}=0.00$ & Accept Ha4 \\
\hline
\end{tabular}

There was no statistically significant association at the 5\% level between gender and reasons for following a gluten-free diet and there was no statistically significant difference at the $5 \%$ level between test scores and education. However, there was a statistically significant difference in test scores between the general public and NCGACs at the 5\% level.

There also was a statistically significant difference between test scores and genders at the 5\% level. The table below illustrates the differences in test scores between genders:

Table 3: Differences in Test Scores between genders in the general population and NCGACs

\begin{tabular}{|l|l|}
\hline Group & Different From \\
\hline Female_GP & Female_NCGAC \\
\hline Female_NCGAC & $\begin{array}{l}\text { Female_GP , Male_GP, } \\
\text { Male_NCGAC }\end{array}$ \\
\hline Male_GP & Female_NCGAC \\
\hline Male_NCGAC & Female_NCGAC \\
\hline
\end{tabular}

The analysis showed that NCGAC women had

the higher overall test scores while men in the general public had lower test scores.

\section{Alpha and Beta Errors}

As Ho2 and Ho4 were rejected, there was the possibility of an alpha error. The p-value for Ho2 and Ho4 were 0.000017 and 0.000006 respectively. The likelihood of an alpha error is small; both p-values are still statistically significant even if the cut off for alpha was decreased from 0.05 to 0.01 .

As Ho1 and Ho3 were accepted, there was the possibility of beta errors. The p-values were 0.43 and 0.70 respectively. As both values were much greater than 0.05 , and the power for the T-test was $99 \%$, the chance of a beta error was low. However, more respondents would decrease the likelihood of it occurring.

\section{Discussion}

Considering the increasing prevalence of gluten-free products and diets, the current level of knowledge among the general public and NCGACs is of concern. The average scores were 3.01 out of 7 (43\%) and 3.92 out of 7 (56\%) for the general public and NCGACs respectively.

Understandably, the NCGACs had a higher product literacy and understanding of gluten, gluten-free products and diets. $41.7 \%$ of NCGACs were aware that gluten-free processed foods can have higher calories and lower nutritional content than their gluten counterparts, while only $15.6 \%$ of the general public were aware of this fact. However, there still seems to be some deficiencies in knowledge as only $27.8 \%$ and $32 \%$ of NCGACs knew that triticale and kamut are grains that contain gluten. The overall percentages do raise concern as they suggest a lack of basic knowledge regarding gluten, as only a low percentage of respondents could correctly identify the grains that contain gluten.

The results in this study (for reasons to partake in a gluten-free diet) are not consistent with the results found from existing studies. This study found that $8 \%$ of all respondents chose to partake in a gluten-free diet as they thought it to be healthier, and $1 \%$ of all respondents did so as they thought it would aid with weight loss.

In contrast, a study conducted by Mintel found that $65 \%$ of consumers elected to partake in a gluten-free diet because they thought it to be healthier, and $27 \%$ of consumers did so because they thought it would aid in 
weight loss efforts (Mintel, 2013). However, this study was limited in its delivery to a diverse group of respondents (please see the Limitations section below).

The results found that NCGAC women had the highest overall tests scores, while men in the general public had the lowest test scores. This trend is consistent with existing literature regarding gender and nutritional knowledge. A study conducted by Nayga in 2000 found that males were less likely to use food labels than females and a study examining Korean college students in 2012 found that women seemed to have a higher nutritional knowledge than males (Nayga, 2000) (Kim, Kim, \& Jung, 2012).

\section{Recommendations}

The average member of the general public seems to have poor product literacy and understanding of gluten. Consumers do not know what products typically contain gluten. There also appears to be misconceptions about the benefits of a gluten-free diet for a non-celiac consumer, as most believed (or did not know) that gluten-free processed foods have higher calories and lower nutritional content than their counterparts. As such, the researcher believes that consumer (both NCGAC and the general public) knowledge of gluten and gluten-free products/diets needs to be increased.

While the labeling requirements of gluten-free products is determined by Health Canada, CFIA is the agency that regulates these claims (including on restaurant menus). This could be an opportunity for EHO's to become involved. Recently, EHO's began enforcing the Trans Fat regulation in BC restaurants to ensure compliance with the trans-fat content of products. As provincial EHO's are mandated to inspect restaurants, it would not be inconceivable for them to also verify gluten-free claims.

\section{Limitations}

The majority of respondents were non-celiac

Asian/Caucasian females aged 20-29 with some form of post-secondary education who do not regularly partake in gluten-free diets. The researcher reached out to his contacts and encouraged them to forward the survey in hopes of obtaining a sufficiently broad population. Unfortunately, this was not achieved and the targeted demographic was not sufficiently reached as the majority of participants stated that they did not follow a gluten-free diet.

Upon reviewing feedback comments on Facebook and SurveyMonkey, the some questions may have been too technical. Also, there could be the possibility that participants randomly selected the correct answers by random selection or from searching the answers on the internet.

\section{Future Research Suggestions}

The researcher suggests that future projects similar to this need to be distributed in such a way that it reaches a much broader audience and the targeted demographic. Conversely, future projects could also be designed to a limited targeted demographic. The study could be specifically targeted to a group, such as employees who work at gluten-free bakeries, stores or manufacturers.

If any knowledge-testing questions are to be asked, they should be phrased much more simply without technical information to not confuse participants.

\section{Conclusion}

While women who elected to participate in gluten-free diets (but did not have CD themselves) had higher overall test scores than men, there still seems to be deficiencies in consumer knowledge regarding gluten, gluten-free products and diets. Furthermore, the results suggest that consumers are unaware of the potential adverse health effects that could result from gluten-free diets.

Knowledge regarding these topics should be increased to reduce consumer deception and potential illnesses from accidental ingestion. 


\section{Acknowledgements}

The author would like to thank the BCIT Environmental

Health Research Advisors for their support and

contributions.

\section{Abbreviations}

CD: Celiac's Disease

NCGS: Non-celiac gluten sensitivity

NCGAC: Non-Celiac Gluten Avoiding Consumers

\section{Competing Interests}

The author declares that they have no competing interest, investment or involvement in the research topic beyond the scope of the ENVH 8410 Applied Research course.

\section{References}

British Columbia Insitute of Technology. (2013). Research Ethics for Human Partipants . Retrieved from Applied Research at BCIT: http://www.bcit.ca/files/pdf/policies/6500.pdf

Cadenhead, K., \& Sweeny, M. (2013). Gluten elimination diets: Facts of patients on this food fad. British Columbia Medical Journal, 55(3), 161-165.

Canadian Celiac Association . (2011a). About Celiac Disease. Retrieved from http://www.celiac.ca/index.php/about-celiacdisease-2/symptoms-treatment-cd/

Canadian Celiac Association. (2011). About Celiac Disease. Retrieved from http://www.celiac.ca/index.php/about-celiacdisease-2/symptoms-treatment-cd/

Canadian Celiac Association. (2011b). Gluten Sensitivity. Retrieved from http://www.celiac.ca/index.php/glutensensitivity/

Codex Alimentarius. (2008). Codex Standard for Special Dietary Use for Persons Intolerant to Gluten. CODEX STAN 118-1979. Rome, Italy. Retrieved from

http://www.codexalimentarius.net/input/downloa d/standards/291/cxs_118e.pdf

Collin, P., Thorell, K., Kaukinen, \& M'aki, M. (2004). The Safe Threshold for Gluten Contamination in Gluten-Free Products: Can Trace Amounts be Accepted in the Treatment of Coeliac Disease? Alimentary Pharmacology and Therapeutics, 19(12), 1277-1283.

Diaz-Amgio, C., \& Popping, B. (2012). Gluten and Gluten-Free: Issues and Considerations of Labeling Regulations, Detection Methods, and
Assay Validation. Journal of AOAC International, 95(2), 337-348.

DiSabatino, A., \& Corazza, G. (2012). Nonceliac Gluten Sensitivity: Sense or Sensability? Annals of INternal Medicine, 156(4), 309-W86.

Facebook. (2014). Facebook. Retrieved from Facebook: www.facebook.com

Food and Drug Regulations (C.R.C. C. 870) (b). (2013). Justice Laws Website. Retrieved from http://laws-

lois.justice.gc.ca/eng/regulations/C.R.C.,_c._870 /page-177.html\#docCont

Food and Drug Regulations (C.R.C., C. 870) (a). (2013). Justice Laws Website. Retrieved from http://laws-

lois.justice.gc.ca/eng/regulations/C.R.C.,_c._870 /page-177.html\#docCont

Gulli, C. (2013). The Dangers of going Gluten-free. Macleans Magazine. Retrieved from http://www2.macleans.ca/2013/09/10/gonegluten-free/

Health Canada. (2012). Health Canada's Position on Gluten-free claims. Retrieved from http://www.hc-sc.gc.ca/fn-an/securit/allerg/celcoe/gluten-position-eng.php

Hintze, J. (2013). NCSS 9. Kaysville, Utah, USA: LLC. Retrieved from www.ncss.com.

Holmes, G. (2013). Non coeliac gluten sensitivity. Gasteroenterology and Hepatology from bed to Bench, 115-119.

Kagnoff, M. (2007). Celiac disease: pathogenesis of a model immunogenetic disease. Journal of Clinical Investigation(117), pp. 41-49. Retrieved from http://www.biomedcentral.com/17417015/10/13

Kim, H. K., Kim, J. H., \& Jung, H. K. (2012). A comparison of health related habits, nutrition knowledge, dietary habits, and blood composition according to gender and weight status of college students in Ulsan. Korean Journal of Nutrition, 45 (4), pp. 336-346.

Lardizabal, A., Lynn, M., Niemann, Sue, L., \& Hefle. (2002). Immunochemical Analysis of Various Foods and Food Ingridents for Detecable Gluten Content: Implications for Wheat-Alleric and Celiac Sprue Patients. Journal of Allergy and Clinical Immunology, 109.

Microsoft Office. (2007). Microsoft Excell . Microsoft Corporation.

Mintel. (2013). More than a quarter of Americans eat gluten-free foods to help them lose weight. Retrieved from http://www.mintel.com/presscentre/food-and-drink/gluten-free-food-to-loseweight

Nayga, R. M. (2000). Nutrition Knowledge, Gender, and Food Label Use. Journal of Consumer Affairs, 34, pp. 97-113.

Roe, S. (2008). Whole Foods Pulls 'Gluten-Free' Products from Shelves after Tribune Story: Report a Month Ago had Found High Levels of Allergen 
in Products. Chicago Tribune. Retrieved from http://articles.chicagotribune.com/2008-1231/news/chi-whole_foodsdec31_1_foodsmarket-whole-foods-libba-letton

Rubio-Tapia, A., Kyle, R., Johnson, D., Erdtmann, F., Brantner, T., Kim, W., . . . Murrary, J. (2009). Increased prevalence and mortality in undiagnosed celiac disease. Gatroenterology. 137, pp. 88-93Sapone, A., Bai, J., Ciacci, C., Dolinsek, J., Green, P., Hadjivassiliou, M., . . . Fasano, A. (2012). Spectrum of gluten-related disorders: consenus on new nomenclature and classification. BCM Med, 10, 10-13. Retrieved from http:/www.biomedcentral.com/17417015/10/13

Sapone, A., Lammers, K., Casolaro, V., Cammartoa, M., Giuliano, M., De Rose, M., . . . Fasano, A. (2011). Divergence of gut permeability and mucosal immune gene expression in two glutenassociated conditions: celiac disease nad gluten sensitivity. BMC Med. Retrieved from http://www.biomedcentral.com/1741-7015/9/23

SurveyMonkey. (2013). SurveyMonkey. Retrieved from SurveyMonkey: https://www.surveymonkey.com/

Tanpowpong, P., Ingham, T., Lampshire, P., Kirchberg, F., Epton, M., Crane, J., \& Camargo, C. (2012). Coeliac Disease and Gluten Avoidance in New Zealand Children. Arch Dis Child(97), 12-16.

Villanacci, V., \& Corazza, G. (2005). Coeliac Disease. Journal of Clinical Pathology(58), pp. 573-574.

Worosz, M., \& Norbert, W. (2012). A Cautionary Tale of Purity, Labeling and Product Literacy in the Gluten--free Market. The Journal of Consumer Affairs, 43(2), pp. 288-318 